\title{
Kasuistiken
}

Hautarzt 2020 $\cdot 71: 1000-1006$

https://doi.org/10.1007/s00105-020-04669-1

Online publiziert: 20 . August 2020

(C) Der/die Autor(en) 2020

\author{
Bijan Koushk-Jalali' · Svenja Schürrle' · Thomas Kuntz' · Georgios Mitrakos' \\ Christian Tigges ${ }^{1}$. Frank Oellig ${ }^{2}$. Andreas Hammacher ${ }^{3} \cdot$ Steffi Silling ${ }^{4}$. \\ Ulrike Wieland $^{4} \cdot$ Alexander Kreuter $^{1}$ \\ ' Klinik für Dermatologie, Venerologie und Allergologie, HELIOS St. Elisabeth Klinik Oberhausen, \\ Universität Witten/Herdecke, Oberhausen, Deutschland \\ ${ }^{2}$ Institut für Pathologie, Mülheim an der Ruhr, Deutschland \\ ${ }^{3}$ Klinik für Mund-, Kiefer- und Gesichtschirurgie, Malteser Krankenhaus St. Johannes-Stift, Duisburg \\ Deutschland \\ ${ }^{4}$ Institut für Virologie, Nationales Referenzzentrum für Papillom- und Polyomaviren, Universität zu Köln, \\ Köln, Deutschland
}

\section{Plattenepithelkarzinom auf dem Boden eines oralen Lichen planus}

\section{Bericht zweier Fälle}

Kaum eine chronisch entzündliche Hauterkrankung hat ein so breites klinisches Spektrum wie der Lichen planus. Bei Beteiligung der Mundschleimhaut imponiert die Erkrankung oft als weißliche, nicht abwischbare Streifung (Wickham-Zeichnung), die im Verlauf auch zu schmerzhaften Erosionen und Ulzerationen führen kann. Die häufigsten oralen Lokalisationen sind Wangenschleimhaut, Zunge und Gingiva [1]. Chronisch aktive Verläufe eines Lichen planus mucosae können zur Entstehung von Karzinomvorläuferläsionen oder invasiven Plattenepithelkarzinomen führen. Demzufolge wird der orale Lichen planus von der Weltgesundheitsorganisation (WHO) auch als fakultative Präkanzerose oder „premalignant condition" eingeordnet [2]. Wir berichten über 2 Fälle von lokal fortgeschrittenen Plattenepithelkarzinomen, die auf dem Boden eines langjährig bestehenden Lichen planus mucosae entstanden sind.

\section{Fallbericht 1}

Ein 60-jähriger Patient stellte sich mit seit 3 Jahren bestehenden, langsam zunehmenden Schmerzen im Bereich der

Alle Autoren hatten vollen Zugang zu den publizierten Daten und tragen die Verantwortung für deren Richtigkeit. Alle Autoren haben bei der Erstellung des Manuskriptes mitgewirkt.
Zunge vor, die v. a. bei Nahrungs- und Flüssigkeitsaufnahme bestanden. Sieben Jahre zuvor sei anamnestisch ein Lichen planus der Mundschleimhaut diagnostiziert worden. Die bisher unregelmäßig erfolgte Lokaltherapie bestand aus der Applikation von Triamcinolonacetonid in Haftsalbe. Dies führte jedoch in den letzten Wochen zu keiner wesentlichen klinischen Besserung. Als Vorerkrankungen bestanden ein Diabetes mellitus Typ 2, arterielle Hypertonie und Vorhofflimmern. Eine aktive oder abgelaufene virale Hepatitis (Hepatitis A, B oder C) lag nicht vor. Übermäßiger Alkoholkonsum und Nikotinabusus wurden verneint. In der dermatologischen Untersuchung imponierten weißliche, zum Teil retikuläre, nicht abstreifbare Plaques an Wangenschleimhaut und Zunge. Zudem zeigten sich an der linken Zungenseite 2 umschriebene Ulzerationen (• Abb. 1). Die weitere Inspektion der Haut zeigte keine auffälligen Befunde, im Genitalbereich fielen jedoch ebenfalls eine retikuläre, weißliche Streifung und außerdem Synechienbildung an Glans penis und Präputium mit beginnender Phimose auf (• Abb. 2).

Zur weiterführenden histopathologischen Abklärung wurden tangentiale Exzisionen der beiden Ulzerationen am Zungenrand vorgenommen. In der histopathologischen Untersuchung zeigten sich 2 subtotal exzidierte, hochdiffe- renzierte, gering verhornende Plattenepithelkarzinome mit einer Tumordicke von 2,4 und $1,9 \mathrm{~mm}$ (• Abb. 3). Die immunhistochemische Färbung für p16INK4a, einem indirekten Marker für Hochrisiko-HPV(humanes Papillomavirus)-Onkogen-Expression, fiel negativ aus (• Abb. 4). Dazu passend war auch die virologische PCR(Polymerasekettenreaktion)-Diagnostik für $38 \mathrm{HPV}$ des Genus Alpha (Hoch- und NiedrigrisikoHPV-Typen) negativ.

Zur weiteren operativen Sanierung der Zungenläsionen wurde der Patient in eine Klinik für Mund-, Kiefer- und Gesichtschirurgie verlegt. Die präoperative Ausbreitungsdiagnostik inklusive Computertomographie von Hals und Thorax, Sonographie des Abdomens und Ösophagogastroduodenoskopie ergab keine pathologischen Befunde. Es erfolgte eine Zungenteilresektion links mit intraoperativer Schnellschnittdiagnostik, die nun ein $1,3 \mathrm{~cm}$ tief in die Muskulatur infiltrierend wachsendes Plattenepithelkarzinom mit Lymphangiosis carcinomatosa, aber ohne Gefäßeinbrüche oder perineurale Tumorausbreitung zeigte. Die Defektrekonstruktion erfolgte mittels eines mikrovaskulär reanastomosierten, perforatorgestielten, rein kutanen Hauttransplantates vom rechten Oberschenkel (• Abb. 5). Zudem wurde eine Neck-Dissection durchgeführt, die keinen weiteren Tumorbefall zeigte. Unter 


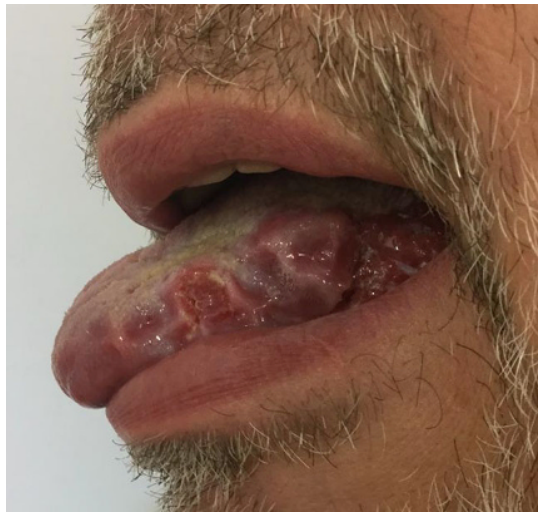

Abb. $1 \Delta$ Es zeigen sich 2 umschriebene Ulzerationen neben einer weißlichen, netzartigen Streifung am Zungenrand links (Fall 1)

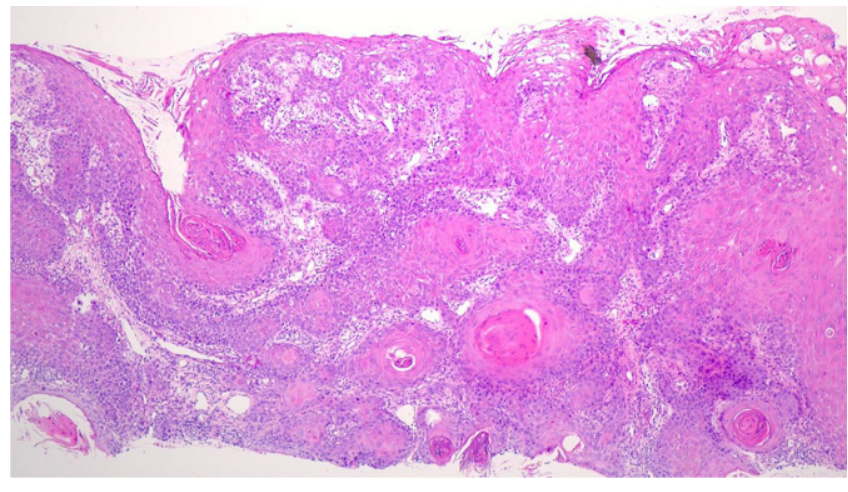

Abb. $3<$ Plattenepithelkarzinom mit Verhornungen, Dyskaryosen sowie hochgradigen Kernatypien (Fall 1; Hämatoxylin-EosinFärbung, Originalvergr. 100:1)

Berücksichtigung der gesamten Befunde handelte es sich somit um ein Zungenkarzinom auf dem Boden eines Lichen planus mucosae mit der Tumorklassifikation pT3, G2, pN0, L1, V0, Pn0, R0. Nach kompletter Einheilung des Transplantates erfolgte eine adjuvante Radiochemotherapie (60 Gy-Strahlentherapie und kombinierte Chemotherapie mit Cisplatin und 5-Fluoruracil); 24 Monate nach Radiochemotherapie war der $\mathrm{Pa}$ tient weiterhin tumorfrei. Aufgrund der durch den genitalen Befall des Lichen planus entstandenen Synechienbildung im Bereich des Penis erfolgte eine Zirkumzision, die komplikationslos verlief.

\section{Fallbericht 2}

Ein 63-jähriger Patient stellte sich mit anamnestisch seit 4 Jahren bestehenden Schleimhautveränderungen bukkal links vor. Vor 1 Jahr sei nach Konsultation eines Zahnarztes eine Probebiopsie erfolgt, die das histopathologische Bild eines Lichen planus mucosae zeigte.
Innerhalb der letzten 3 Monate sei es $\mathrm{zu}$ einer deutlichen Größenzunahme, vermehrten Schmerzen und Blutungen gekommen. Seit 3 Wochen bemerkte der Patient zudem eine Schwellung der Halslymphknoten. Eine Behandlung der Schleimhautläsionen war bisher nicht erfolgt. Als Vorerkrankungen bestanden eine arterielle Hypertonie sowie Zustand nach 2-maligem Apoplex ohne bleibende neurologische Beeinträchtigungen. Eine Virushepatitis (Hepatitis A, B oder C) lag nicht vor. Alkoholkonsum bestand nicht, der Patient war jedoch starker Raucher (20 Packungs-Jahre). In der dermatologischen Untersuchung zeigte sich bukkal links ein tastbarer, derber, ca. $4 \mathrm{~cm}$ durchmessender Tumor mit zerklüfteter, zum Teil ulzerierter Oberfläche und weißlicher Streifung im Randbereich (• Abb. 6). Zur histopathologischen Abklärung erfolgte eine Exzisionsbiopsie aus dem zentralen Anteil des Tumors. Hierbei zeigte sich ein hochdifferenziertes verhornendes Plattenepithelkarzinom mit bis in die
Muskulatur reichenden Tumorausläufern (• Abb. 7). Die immunhistochemische Färbung für p16INK4a sowie der HPV-DNA(Desoxyribonukleinsäure)-

Nachweis mittels PCR (38 HPV-Typen) fielen negativ aus (• Abb. 8). Eine Stanzbiopsie aus der weißlichen Streifung im Randbereich des Tumors zeigte das klassische Bild eines Lichen planus mucosae (• Abb. 9). Zur weiterführenden Diagnostik erfolgte eine Magnetresonanztomographie des Halses. Hier fielen neben der Raumforderung der Wangenschleimhaut links 2 bis zu $18 \mathrm{~mm}$ durchmessende Lymphknoten links submandibulär auf. Die restliche Ausbreitungsdiagnostik war unauffällig. Zur weiteren operativen Sanierung erfolgte die Verlegung in eine Klinik für Mund-, Kiefer- und Gesichtschirurgie. Hier wurde eine radikale Resektion des insgesamt $3 \mathrm{~cm}$ durchmessenden Tumors am Planum buccale links mit Unterkieferkastenresektion links, Osteotomie sowie Defektdeckung mittels Bichat-Lappen vorgenommen. Die komplettierende Neck-Dissection zeigte in 24 entnommenen Lymphknoten $2 \mathrm{Me}$ tastasen. Somit handelte es sich um ein Plattenepithelkarzinom der Mundhöhle auf dem Boden eines Lichen planus mucosae mit der Tumorklassifikation pT2, G2, pN1 (2/24), L0, V0, Pn0, R0. Es erfolgte eine adjuvante Radiatio mit einer Gesamtdosis von 60 Gy. Fünf Jahre nach abgeschlossener Radiatio ist der Patient weiterhin tumorfrei.

\section{Diskussion}

Der Lichen planus ist eine chronisch inflammatorische, immunologisch induzierte mukokutane Dermatose, die vermutlich durch gegen basale Keratinozyten gerichtete CD8-positive T-Zellen verursacht wird [3]. Diverse Triggerfaktoren wie bakterielle oder virale Infektionen, Medikamente oder physikalische Reize (z.B. Morsicatio, Zahnbehandlungen oder Zahnprothesen) werden bei der Entstehung des Lichen planus diskutiert. Auch Tabakrauch wird als irritierende Noxe diskutiert. Der Lichen planus mucosae manifestiert sich am häufigsten in der Mundhöhle. Nach Andreasen werden 6 verschiedene Formen 
unterschieden: retikulär, erosiv, papulös, plaqueförmig, bullös und atrophisch [4]. Die häufigsten Varianten sind dabei die retikuläre und die erosive Form [5]. Eine große retrospektive Studie mit 518 Patienten zeigte, dass der orale Lichen planus am häufigsten bukkal $(87,8 \%)$ und im Bereich der Zunge (56,9\%) auftritt [6]. Der Lichen planus mucosae kann sich jedoch auch im Larynx, im Ösophagus oder in der Genitalregion manifestieren. Der Anteil der an Lichen planus mucosae erkrankten Personen in der Bevölkerung wird auf ca. $0,5-2 \%$ geschätzt, wobei Frauen doppelt so häufig betroffen sind wie Männer [7].

Der Lichen planus mucosae wird zu den fakultativen Präkanzerosen gezählt, die maligne Transformationsrate ist aber gering. In einer kürzlich publizierten $\mathrm{Me}$ taanalyse wurden insgesamt $12.838 \mathrm{~Pa}$ tienten mit oralem Lichen planus aus 33 Studien ausgewertet und eine Progressionsrate zum Plattenepithelkarzinom von 1,2\% ermittelt. Das Risiko der malignen Transformation ist bei Patienten mit oralem Lichen planus, die rauchen (Odds Ratio = 4,62), vermehrt Alkohol konsumieren (Odds Ratio=3,22) oder eine chronische Hepatitis-C-Infektion haben (Odds Ratio=3,77) signifikant erhöht [8].

Mundhöhlenkarzinome, wie in unseren beiden Fällen dargestellt, sind der häufigste Tumortyp unter den KopfHals-Tumoren. Die geschätzte Anzahl an Neuerkrankungen liegt weltweit bei ca. 200.000 Fällen pro Jahr [9]. Mindestens ein Drittel aller oropharyngealen Karzinome ist HPV-induziert. Der Anteil der HPV-induzierten Mundhöhlenkarzinome ist mit 2,2\% deutlich geringer [9]. Die Assoziation von HPV mit oralem Lichen planus ist noch nicht abschließend geklärt. In einer Metaanalyse aus 22 Studien mit insgesamt 835 Patienten, die unter einem oralen Lichen planus litten, konnte eine HPV-Infektion in $35 \%$ der Fälle (Odds Ratio= 6,83) nachgewiesen werden. Die Assoziation von HPV mit oralem Lichen planus scheint dabei starken geografischen Schwankungen $\mathrm{zu}$ unterliegen (Odds Ratio $=5,15$ in Europa und Odds Ratio $=96,6$ in Nordamerika) [10]. Interessanterweise scheint die HPV-Assoziation beim ero-

Hautarzt 2020 $71: 1000-1006$ https://doi.org/10.1007/s00105-020-04669-1

(c) Der/die Autor(en) 2020

B. Koushk-Jalali · S. Schürrle · T. Kuntz · G. Mitrakos · C. Tigges · F. Oellig · A. Hammacher ·
S. Silling · U. Wieland · A. Kreuter

Plattenepithelkarzinom auf dem Boden eines oralen Lichen planus. Bericht zweier Fälle

\section{Zusammenfassung}

Beim Lichen planus handelt es sich vermutlich um eine chronisch inflammatorische, immunologisch induzierte mukokutane Dermatose. Der Lichen planus mucosae manifestiert sich am häufigsten in der Mundhöhle. Diverse Triggerfaktoren wie bakterielle oder virale Infektionen, Medikamente oder physikalische Reize werden bei der Entstehung der Erkrankung diskutiert. Auch eine Assoziation mit Infektionen durch humane Papillomviren wurde beschrieben, ein kausaler Zusammenhang ist jedoch nicht hinreichend belegt. Als fakultative Präkanzerose kann sich auf dem Boden eines Lichen planus mucosae ein Plattenepithelkarzinom entwickeln, die maligne Transformationsrate ist aber gering. Das Risiko der malignen Transformation ist signifikant erhöht bei Patienten mit oralem Lichen planus, die rauchen, vermehrt Alkohol konsumieren oder an Hepatitis C erkrankt sind. Wir beschreiben 2 Patienten, bei denen sich lokal fortgeschrittene Plattenepithelkarzinome auf dem Boden eines langjährig bestehenden oralen Lichen planus entwickelten. Beide Fälle wurden erfolgreich durch radikale Tumorresektion mit anschließender Rekonstruktion und adjuvanter Radiatio/Radiochemotherapie behandelt.

Schlüsselwörter

Lichen planus mucosae · Oraler Lichen ruber · Fakultative Präkanzerose · Mukositis . Transformationsrate

\section{Squamous cell carcinoma arising in oral lichen planus. Report of two cases}

\section{Abstract}

Lichen planus is a chronic inflammatory, immunologically mediated mucocutaneous dermatosis. Lichen planus mucosae predominantly affects the oral cavity. Various trigger factors such as bacterial or viral infections, drugs or physical stimuli are discussed in the development of the disease. An association with human papillomavirus infections has also been described, but is not sufficiently proven. Lichen planus mucosae is considered as a premalignant condition, but the malignant transformation rate is low. The risk of malignant transformation is significantly increased in patients with oral lichen planus who smoke, drink alcohol or have hepatitis C. We describe two patients with locally advanced squamous cell carcinoma that developed on a longstanding oral lichen planus. Both cases were successfully treated with radical tumor resection, subsequent tissue reconstruction, and adjuvant radiation/radiochemotherapy.

\section{Keywords}

Mucosal lichen planus · Oral lichen ruber . Premalignant condition - Mucositis . Transformation rate siven Lichen planus höher $\mathrm{zu}$ sein als bei nichterosiven Formen (Odds Ratio 9,34 vs. 4,32). Im Gegensatz dazu konnte in einer kürzlich publizierten Studie aus Spanien mit 83 prämalignen oralen Läsionen (41 davon oraler Lichen planus) nur in 4 Fällen (4,8\%) HPV-DNA nachgewiesen werden [11].

P16INK4a, ein indirekter Marker für HPV-Onkogen-Expression, wird in der überwiegenden AnzahlHPV-induzierter Dysplasien und Karzinome exprimiert. Zudem ist p16INK4a ein gut etablierter, validierter und prognostisch relevanter Biomarker bei Kopf-Hals-Tumoren [12]. In proliferierenden Geweben zahlreicher Tumorerkrankungen und auch bei chronisch entzündlichen Läsionen wird p16INK4a jedoch auch unabhängig von HPV exprimiert. So konnte auch beim oralen Lichen planus in einer Studie in mehr als $60 \%$ der Fälle eine Überexpression von p16INK4a gezeigt werden [13]. In einer anderen Studie, in der 35 Fälle von oralem Lichen planus bezüglich p16INK4a und HPV-DNA untersucht 


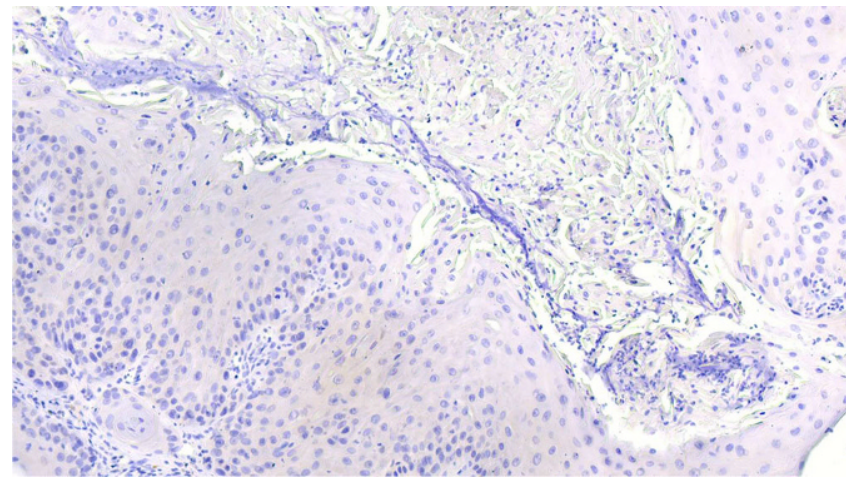

Abb. $4<$ Immunhistochemische Färbung auf p16INK4a. Es zeigen sich nur sehr wenige, fokal p16-positive Zellen. Damit kann die Färbung als negativ gewertet werden (Fall 1; p16INK4a, Originalvergr. 100:1)

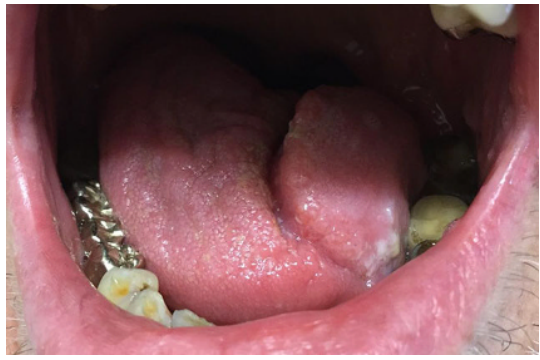

Abb. 5 $\Delta$ Zustand nach Defektrekonstruktion mittels eines mikrovaskulär reanastomosierten, perforatorgestielten, rein kutanen Hauttransplantates vom rechten Oberschenkel (Fall 1)

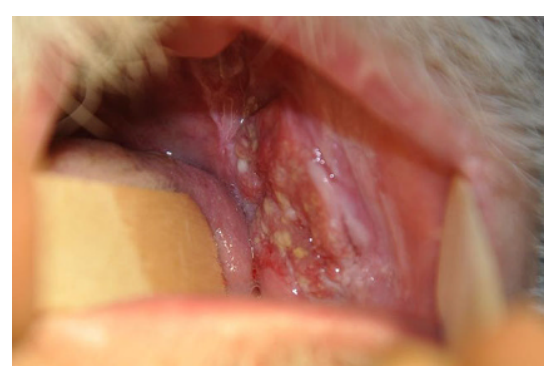

Abb. $6 \Delta$ Etwa $4 \mathrm{~cm}$ durchmessender, derb tastbarer Tumor bukkal links mit zerklüfteter, zum Teil ulzerierter Oberfläche und weißlicher Streifung im Randbereich (Fall 2)

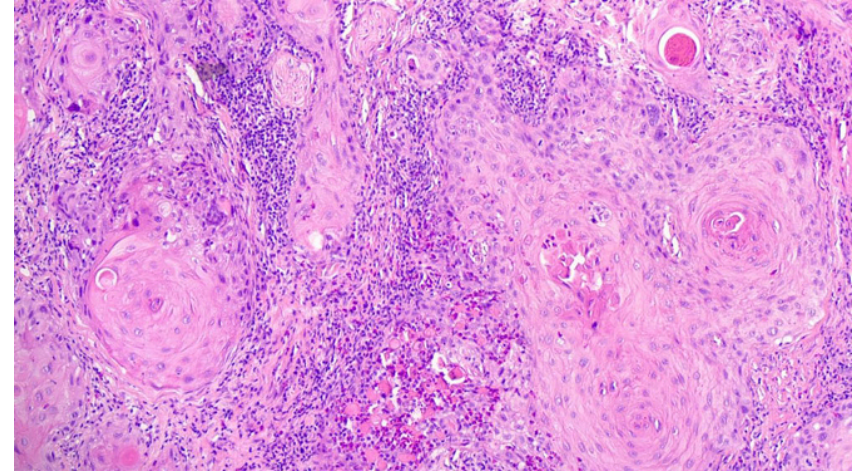

Abb. 7 ॥ Hochdifferenziertes, verhornendes Plattenepithelkarzinom der Wangenschleimhaut. Tumorausläufer erreichen die Präparatränder und die Absetzungslinie in der Tiefe. Sie schieben sich dabe zwischen quergestreifte Muskulatur vor (Fall 2; Hämatoxylin-Eosin-Färbung, Originalvergr. 100:1)

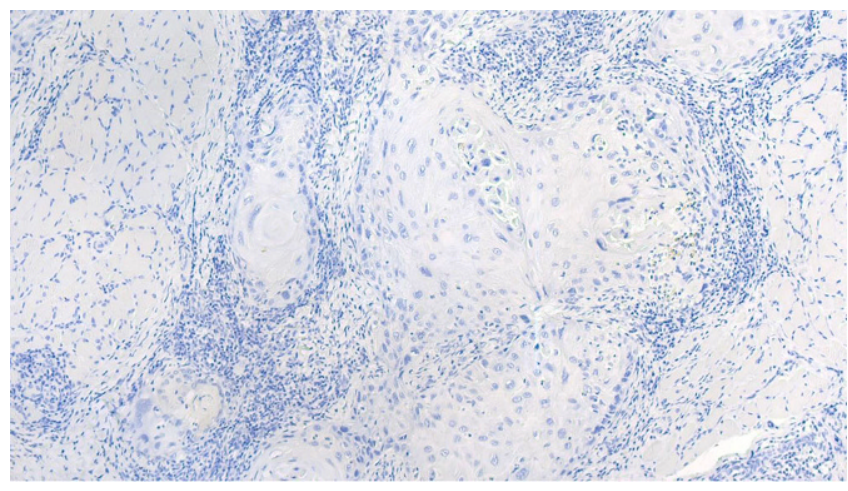

Abb. $8 \Delta$ Immunhistochemische Färbung auf p16INK4a. Diese fiel negativ aus (Fall 2; p16INK4a, Originalvergr. 100:1)

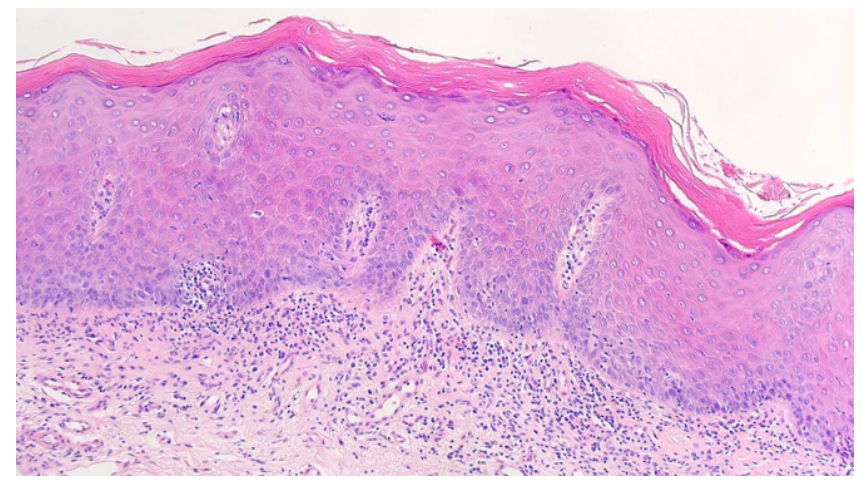

Abb. $9 \Delta$ Histologie aus der Wangenschleimhaut des Randbereichs des Tumors. Es zeigen sich eine Akanthose und plumpe Hyperpapillomatose der Epidermis. Im Papillarkörper imponiert ein lichenoides Entzündungsinfiltrat passend zum Lichen planus mucosae (Fall 2; Hämatoxylin-Eosin-Färbung, Originalvergr. 100:1) wurden, konnte allerdings in lediglich 4 Läsionen HPV nachgewiesen werden [14].

Seit längerer Zeit ist bekannt, dass eine Infektion mit dem Hepatitis-C-Virus ein signifikant erhöhtes Risiko für die Entwicklung eines Lichen planus darstellt [15]. Durch die seit 2014 verfügbaren Kombinationstherapien, bestehend aus Protease-, Polymerase- und NS5AHemmern, ist Hepatitis $C$ heutzutage fast immer heilbar. Ein kürzlich veröffentlichter Review-Artikel hat unterschiedliche klinische Verläufe (81,8\% Abheilung/ Besserung und 18,1\% Verschlechterung/ Persistenz) beim Hepatitis-C-assoziierten Lichen planus nach erfolgreicher Hepatitistherapie gezeigt. Die Wahrschein- lichkeit einer kompletten Abheilung nach antiviraler Therapie war beim oralen Lichen planus am höchsten [16]. In den von uns präsentierten Fällen konnte jedoch eine Hepatitis als Triggerfaktor des oralen Lichen planus ausgeschlossen werden.

Alle kurativ resektablen Mundhöhlenkarzinome sollten, wenn es der Allgemeinzustand des Patienten zulässt, 
operativ behandelt werden. Bei fortgeschrittener T-Kategorie (T3/T4), knappen oder positiven Resektionsrändern, perineuraler Invasion, Gefäßinvasion und/oder Lymphknotenbefall sollte laut AWMF (Arbeitsgemeinschaft der Wissenschaftlichen Medizinischen Fachgesellschaften e. V.) S3-Leitlinie eine postoperative Radio- oder Radiochemotherapie erfolgen [17]. Dieses Vorgehen war auch bei unseren Fällen erfolgreich. Bei ca. $20 \%$ der Patienten kommt es allerdings $\mathrm{zu}$ einem Lokalrezidiv des Mundhöhlenkarzinoms, am häufigsten innerhalb der ersten 2 Jahre. Unsere beiden Patienten blieben im Nachbeobachtungszeitraum von 2 bzw. 5 Jahren tumorfrei.

Bei fortgeschrittenen, rezidivierten oder metastasierten Mundhöhlenkarzinomen wird bei Patienten in gutem Allgemeinzustand in der Palliativsituation eine Kombination aus platinhaltiger Chemotherapie (vorzugsweise Cisplatin) und 5-Fluorouracil (ExtremeSchema) sowie dem „Epidermal-growthfactor-Rezeptor-Antikörper" Cetuximab vorgeschlagen (https://www.awmf.org/ leitlinien/detail/anmeldung/1/1l/007-

100OL.html, Konsultationsfassung S3Leitlinie Diagnostik und Therapie des Mundhöhlenkarzinoms). Für eine lange Zeit existierte kein Therapiestandard nach Progression unter bzw. nach der platinhaltigen Erstlinientherapie.

Seit Ende des Jahres 2016 besteht jedoch eine Zulassung der FDA (Food and Drug Administration) für den Checkpoint-Inhibitor Nivolumab bei Plattenepithelkarzinomen des Kopfes und Halses nach erfolgter platinbasierter Chemotherapie (Checkmate 141). Mitte des Jahres 2019 folgte die FDAZulassung von Pembrolizumab als Erstlinienbehandlung (Keynote 048) [18]. Obwohl unseres Wissens bisher noch nicht über den Einsatz von CheckpointInhibitoren bei Plattenepithelkarzinomen auf dem Boden eines oralen Lichen planus berichtet wurde, sollte berücksichtigt werden, dass unter Nivolumab und Pembrolizumab auch Lichen-planus-artige Hautveränderungen beschrieben wurden und sich somit ein Lichen planus unter Checkpoint-Inhibition ver- schlechtern bzw. exazerbieren könnte [19].

Zusammenfassend verdeutlichen die hier dargestellten und ähnliche Fallberichte aus der Literatur, dass auf dem Boden eines chronisch aktiven mukosalen Lichen planus Plattenepithelkarzinome entstehen können [7, 20]. Obwohl das Risiko einer malignen Transformation insgesamt niedrig ist und in lediglich ca. $1 \%$ aller Fälle auftritt, sollten bei Patienten mit mukosalem Lichen planus auch nach erfolgter Therapie regelmäßige klinische Kontrolluntersuchungen erfolgen.

\section{Fazit für die Praxis}

- Beim chronisch aktiven oralen Lichen planus besteht ein geringes, aber dennoch erhöhtes Risiko für die Entstehung eines Plattenepithelkarzinoms.

- Die WHO (Weltgesundheitsorganisation) ordnet den oralen Lichen planus als fakultative Präkanzerose bzw. „premalignant condition“ ein.

- Eine Assoziation mit humanen Papillomviren ist beim oralen Lichen planus beschrieben, jedoch nicht gesichert.

- Patienten mit oralem Lichen planus sollten in regelmäßigen Abständen klinisch untersucht werden, um eine maligne Transformation frühestmöglich zu erkennen.

- Resektable Mundhöhlenkarzinome sollten primär operativ behandelt werden.

- Auch lokal fortgeschrittenere hochdifferenzierte Karzinome auf dem Boden eines oralen Lichen planus können kurativ behandelt werden.

\section{Korrespondenzadresse}

\section{Prof. Dr. med. Alexander Kreuter}

Klinik für Dermatologie, Venerologie und Allergologie, HELIOS St. Elisabeth Klinik Oberhausen, Universität Witten/Herdecke Josefstr. 3, 46045 Oberhausen, Deutschland Alexander.Kreuter@helios-gesundheit.de

Funding. Open Access funding provided by Projekt DEAL.
Einhaltung ethischer Richtlinien

Interessenkonflikt. B. Koushk-Jalali, S. Schürrle, T. Kuntz, G. Mitrakos, C. Tigges, F. Oellig, A. Hammacher, S. Silling, U. Wieland und A. Kreuter geben an, dass kein Interessenkonflikt besteht.

Für diesen Beitrag wurden von den Autoren keine Studien an Menschen oder Tieren durchgeführt. Für die aufgeführten Studien gelten die jeweils dort angegebenen ethischen Richtlinien. Für Bildmaterial oder anderweitige Angaben innerhalb des Manuskripts, über die Patienten zu identifizieren sind, liegt von ihnen und/oder ihren gesetzlichen Vertretern eine schriftliche Einwilligung vor.

Open Access Dieser Artikel wird unter der Creative Commons Namensnennung 4.0 International Lizenz veröffentlicht, welche die Nutzung, Vervielfältigung, Bearbeitung, Verbreitung und Wiedergabe in jeglichem Medium und Format erlaubt, sofern Sie den/die ursprünglichen Autor(en) und die Quelle ordnungsgemäß nennen, einen Link zur Creative Commons Lizenz beifügen und angeben, ob Änderungen vorgenommen wurden.

Die in diesem Artikel enthaltenen Bilder und sonstiges Drittmaterial unterliegen ebenfalls der genannten Creative Commons Lizenz, sofern sich aus der Abbildungslegende nichts anderes ergibt. Sofern das betreffende Material nicht unter der genannten Creative Commons Lizenz steht und die betreffende Handlung nicht nach gesetzlichen Vorschriften erlaubt ist, ist für die oben aufgeführten Weiterverwendungen des Materials die Einwilligung des jeweiligen Rechteinhabers einzuholen.

Weitere Details zur Lizenz entnehmen Sie bitte der Lizenzinformation auf http://creativecommons.org/ licenses/by/4.0/deed.de.

\section{Literatur}

1. Gümrü B (2013) A retrospective study of 370 patients with oral lichen planus in Turkey. Med Oral Patol Oral Cir Bucal 18:e427-e432

2. Barnes L, Eveson JW, Reichart P, Sidransky D (2005) Pathology and genetics of head and neck tumours. WHO Classification of Tumours, 3. Aufl. Bd. 9, S163-175

3. Wagner G, Rose C, Sachse MM (2013) Clinical variants of lichen planus. J Dtsch Dermatol Ges 11(4):309-319

4. Andreasen JO (1968) Oral lichen planus. A clinical evaluation of 115 cases. Oral Surg Oral Med Oral Pathol 25(1):31-42

5. Boyd AS, Neldner KH (1991) Lichen planus. J Am Acad Dermatol 25(4):593-619

6. Shen ZY, Liu W, Zhu LK, Feng JQ, Tang GY, Zhou ZT (2012) A retrospective clinicopathological study on oral lichen planus and malignant transformation: analysis of 518 cases. Med Oral Patol Oral Cir Bucal 17(6):e943-e947

7. Hilgers M, Megahed M (2014) Oral lichen planus. Insight into the pathogenesis and therapeutic options. Hautarzt 65(5):393-395

8. Idrees M, Kujan O, Shearston K, Farah CS (2020) Oral lichen planus has a very low malignant transformation rate: a systematic review and meta-analysis using strict diagnostic and inclusion 
criteria. J Oral Pathol Med. https://doi.org/10. 1111/jop.12996

9. de Martel C, Plummer M, Vignat J, Franceschi S (2017) Worldwide burden of cancer attributable to HPV by site, country and HPV type. Int J Cancer 141(4):664-670

10. Ma J, Zhang J, Zhang Y, Lv T, Liu J (2016) The magnitude of the association between human papillomavirus and oral lichen planus: a metaanalysis. PLoSONE 11(8):e161339

11. Gomez-Armayones S, Chimenos-Küstner E, Marí A etal (2019) Human papillomavirus in premalignant oral lesions: no evidence of association in a Spanish cohort. PLoSONE 14(1):e210070

12. Hsieh JC, Wang HM, Wu MH et al (2019) Review of emerging biomarkers in head and neck squamous cell carcinoma in the era of immunotherapy and targeted therapy. Head Neck 41 (Suppl 1):19-45

13. Montebugnoli L, Venturi M, Gissi DB, Leonardi E, Farnedi A, Foschini MP (2011) Immunohistochemical expression of p16(INK4 A) protein in oral lichen planus. Oral Surg Oral Med Oral Pathol Oral Radiol Endod 112(2):222-227

14. Montebugnoli L, Gissi DB, Scapoli L et al (2014) p16(INK4) expression is not associated with human papillomavirus in oral lichen planus. Oral Surg Oral Med Oral Pathol Oral Radiol 118(6):694-702

15. Shengyuan $L$, Songpo $Y$, Wen $W$, Wenjing $T$, Haitao Z, Binyou W (2009) Hepatitis C virus and lichen planus: a reciprocal association determined by a meta-analysis. Arch Dermatol 145(9):1040-1047

16. Barajas L, Steuer M, Amerson E (2020) Variable clinical course of lichen planus following hepatitis $C$ cure with direct-acting antivirals: a case series and literature review. J Am Acad Dermatol. https:// doi.org/10.1016/j.jaad.2020.05.159 (Online im Druck)

17. Wolff KD, Follmann M, Nast A (2012) The diagnosis and treatment of oral cavity cancer. Dtsch Arztebl Int 109(48):829-835

18. Le X, Ferrarotto R, Wise-Draper T, Gillison M (2000) Evolving role of immunotherapy in recurrent metastatic head and neck cancer. J Natl Compr Canc Netw 18(7):899-906

19. De Lorenzi C, André R, Vuilleumier A, Kaya G, Abosaleh M (2020) Bullous lichen planus and antiprogrammed cell death-1 therapy: case report and literature review. Ann Dermatol Venereol 147(3):221-227

20. Thiessen N, Alter M, Kapp A, Gutzmer R (2013) Squamous cell carcinoma developing in oral lichen planus. Hautarzt 64(11):843-845

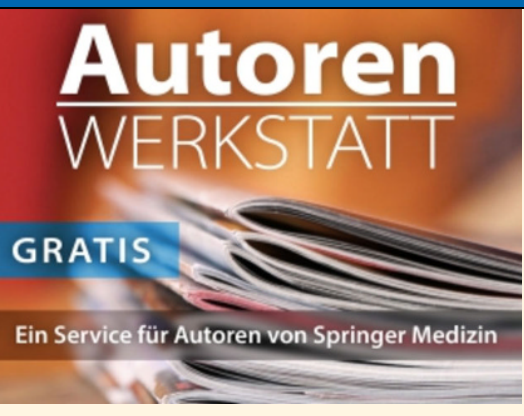

\section{Fortbildungen für Autoren und Gutachter}

Die ersten Veröffentlichungen sind für jeden Mediziner ein wichtiger Schritt in seiner Karriere als Wissenschaftler. Wissenschaftliche Artikel sind entscheidend dafür, dass die eigene Arbeit in der Community wahrgenommen wird. Es geht darum, die eigenen Ideen national und international auszutauschen und sicherzustellen, dass die Ergebnisse Wirkung erzielen.

Die Online-Kurse der Autorenwerkstatt helfen, sich leicht einen Überblick über das Schreiben, Einreichen, Begutachten und Veröffentlichen eines Manuskripts zu verschaffen.

5 Online-Kurse zu den wichtigsten Standards des wissenschaftlichen Publizierens:

- Wie verfasse ich ein Manuskript?

- Writing in English für deutschsprachige Autoren

- Wie funktionieren Publikation und Begutachtung?

- Anleitung zur Open-Access-Veröffentlichung

- Leitfaden zur Peer-Review-Begutachtung

Für alle, die auf SpringerMedizin.de registriert sind!

Jetzt gratis fortbilden unter

www.springermedizin.de/autorenwerkstatt/ 
Hier steht eine Anzeige.

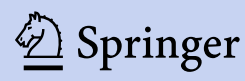

